\title{
A simple catalytic synthesis of condensed pyridones from $o$-bromoarylcarboxamides via palladacycles and ipso substitution
}

Raffaella Ferraccioli,* Davide Carenzi, Elena Motti, and Marta Catellani*

CNR-Istituto di Scienze e Tecnologie Molecolari (ISTM), Dipartimento di Chimica Organica e Industriale, Università di Milano, Via C. Golgi 19, 20133 Milano, Italy and Dipartimento di Chimica Organica e Industriale, Università di Parma, Parco Area delle Scienze, 17/A, 43100 Parma, Italy

\section{Supporting Information}

General Considerations: Unless otherwise specified, all reagents and solvents were purchased from commercial suppliers and used without further purification. ${ }^{1}$ 2-Bromo- $N$-methylbenzamide $\mathbf{2 a}^{2}$, 3-bromothiophene-2-carboxylic acid methylamide $\mathbf{2 b},{ }^{3}$ 3-bromobenzo[b]thiophene-2carboxylic acid methylamide $\mathbf{2 c}{ }^{3}$ were prepared according to reported procedures. The palladiumcatalyzed reactions were run under nitrogen using standard Schlenk and vacuum line techniques. Melting points are uncorrected. Unless otherwise indicated ${ }^{1} \mathrm{H}$ and ${ }^{13} \mathrm{C}$ NMR spectra of 2 and $\mathbf{1}$ were recorded in $\mathrm{CDCl}_{3}$ or DMSO- $d_{6}$ on Bruker 300-AMX and AVANCE300 spectrometers at 300 and $75 \mathrm{MHz}$, respectively. The reported ${ }^{1} \mathrm{H}$ NMR data of compounds 2 were obtained after treatment with $\mathrm{D}_{2} \mathrm{O}$. E. I. mass spectra were determined on a VG Analytical 7070 EQ mass spectrometer with an attached VG Analytical 11/250 data system. IR spectra were recorded with a Perkin-Elmer FT-IR 1725X spectrophotometer.

Synthesis of 12, 13.
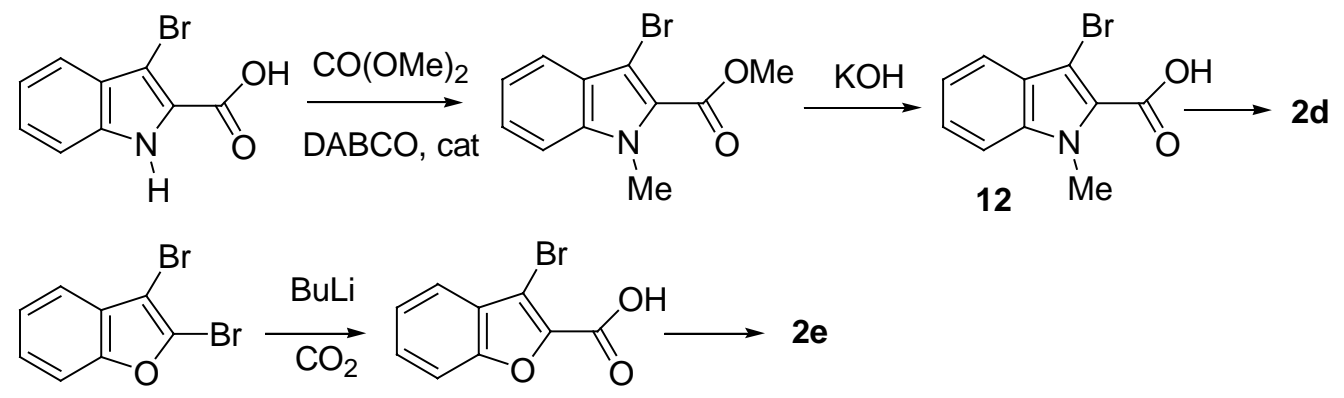

13

Compound 13. A solution of 3-bromo- $1 H$-indole-2-carboxylic acid ${ }^{4}$ (1.53 g, 6.38 mmol), 1,4diazabicyclo[2.2.2]octane (DABCO) (0.86 g, $7.65 \mathrm{mmol})$, anhydrous DMF (1.5 mL) in dimethyl carbonate $(15 \mathrm{~mL})$ was heated at $95{ }^{\circ} \mathrm{C}$ for $24 \mathrm{~h}$ under stirring, then cooled to room temperature. The reaction mixture was diluted with AcOEt $(50 \mathrm{~mL})$, washed with $\mathrm{H}_{2} \mathrm{O}(100 \mathrm{~mL})$, twice with $10 \%$ aq. citric acid $(50 \mathrm{~mL})$, and $\mathrm{H}_{2} \mathrm{O}(50 \mathrm{~mL})$. The organic phase was separated, dried over $\mathrm{Na}_{2} \mathrm{SO}_{4}$ and evaporated under reduced pressure to give a crude oily residue containing 3-bromo-1methyl-1H-indole-2-carboxylic acid methyl ester which was used without purification. 1N KOH (15 
$\mathrm{mL})$ was added dropwise to a suspension of the crude Br-ester $(1.0 \mathrm{~g}, 3.73 \mathrm{mmol})$ in $\mathrm{MeOH}(40$ $\mathrm{mL}$ ). The mixture was heated at reflux for $1 \mathrm{~h}$, then cooled to room temperature and concentrated in vacuo. The residue was treated with $1 \mathrm{~N} \mathrm{HCl}(50 \mathrm{~mL})$ and the aqueous layer was extracted with AcOEt $(2 \times 50 \mathrm{~mL})$. The combined organic phase was dried over $\mathrm{Na}_{2} \mathrm{SO}_{4}$, and evaporated under reduced pressure to afford compound $\mathbf{1 2}$ as a solid (Yield: $0.76 \mathrm{mg}, 80 \%$ ). A sample was purified by crystallization (acetone $\left./ \mathrm{H}_{2} \mathrm{O}, 1 / 1\right) \mathrm{mp} 179{ }^{\circ} \mathrm{C}$ with decomposition, (lit ${ }^{5} \mathrm{mp} 180{ }^{\circ} \mathrm{C}$ ).

Compound 13. To a solution of 2,3-dibromo-benzofuran ${ }^{6}(0.90 \mathrm{~g}, 3.26 \mathrm{mmol})$ in anhydrous THF $(10 \mathrm{~mL})$, cooled to $-78^{\circ} \mathrm{C}$ was added dropwise BuLi (2.24 $\mathrm{mL}$ of $1.6 \mathrm{M}$ in hexane) under nitrogen. The resulting solution was stirred $30 \mathrm{~min}$ at $-78^{\circ} \mathrm{C}$, then carbon dioxide $\left(\mathrm{CO}_{2}\right)$ was bubbled into the mixture for $1 \mathrm{~h}$. The reaction mixture was allowed to warm to room temperature and $\mathrm{CO}_{2}$ bubbling was continued for additional $1 \mathrm{~h}$. The reaction was quenched with $\mathrm{H}_{2} \mathrm{O}(20 \mathrm{~mL})$. The solution was acidified with $1 \mathrm{~N} \mathrm{HCl}$. The precipitate was filtered and dried to afford compound $13(0.60 \mathrm{~g}, 77 \%$ yield). ${ }^{1} \mathrm{H}$ NMR (DMSO- $\left.d_{6}, 200 \mathrm{MHz}\right): 7.47$ (t, $\left.J=8.0 \mathrm{~Hz}, 1 \mathrm{H}\right), 7.62(\mathrm{dt}, J=8.3,1.4 \mathrm{~Hz}, 1 \mathrm{H}), 7.68$ $(\mathrm{d}, J=8.6 \mathrm{~Hz}, 1 \mathrm{H}), 7.77$ (d, $J=8.3 \mathrm{~Hz}, 1 \mathrm{H}), 14.0$ (bs, 1H). A sample was crystallized: mp 206-207 ${ }^{\circ} \mathrm{C}$, acetone/H2O 1/1, (lit. ${ }^{7} \mathrm{mp} 209{ }^{\circ} \mathrm{C}$ ).

Synthesis of 2. A suspension of the crude bromo-acid $(2.52 \mathrm{mmol})$, a catalytic amount of anhydrous DMF (mmol, $30 \mu \mathrm{L}$ ) and thionyl chloride (oxalyl chloride for $\mathbf{2 d}$ ) (7.56 mmol) in dry $\mathrm{CH}_{2} \mathrm{Cl}_{2}(50 \mathrm{~mL})$ was heated at reflux under stirring for $3 \mathrm{~h}$ (for $\mathbf{2 d}$ the mixture was stirred at room temperature for $1 \mathrm{~h}$, then at reflux for $1 \mathrm{~h}$ ). The solution was cooled to room temperature and concentrated in vacuo. The residue was dissolved in anhydrous $\mathrm{CH}_{2} \mathrm{Cl}_{2}(20 \mathrm{~mL})$ and added dropwise to a solution of the amine $(5.54 \mathrm{mmol}$ ) (for $3 \mathbf{c}: 2.52 \mathrm{mmol}$ of the amine and 2.6 of anhydrous TEA) in $\mathrm{CH}_{2} \mathrm{Cl}_{2}(20 \mathrm{~mL})$ cooled to $0^{\circ} \mathrm{C}$ under stirring. The reaction mixture was allowed to warm to room temperature and stirring was continued for $2 \mathrm{~h}$. After treatment with saturated aq. $\mathrm{NaHCO}_{3}(2 \times 50 \mathrm{~mL})$ and separation of the organic phase, the aqueous layer was extracted with AcOEt $(2 \times 50 \mathrm{~mL})$. The combined organic extracts were dried over $\mathrm{Na}_{2} \mathrm{SO}_{4}$ and evaporated under reduced pressure. The crude residue was purified by chromatography on silica gel (PE/AcOEt).

\section{3-Bromo-1-metil-1H-indole-2-carboxylic acid methylamide (2d).}

Yield: 90\%. M.p.: 140-141 ${ }^{\circ} \mathrm{C}(\mathrm{AcOEt} / \mathrm{PE}, 9 / 1) .{ }^{1} \mathrm{H}$ NMR $\left(\mathrm{CDCl}_{3} / \mathrm{D}_{2} \mathrm{O}\right): \delta 3.06(\mathrm{~s}, 3 \mathrm{H}), 4.05$ (s, $3 \mathrm{H}), 7.22-7.29(\mathrm{~m}, 1 \mathrm{H}), 7.38-7.41(\mathrm{~m}, 2 \mathrm{H}), 7.62(\mathrm{~d}, J=8.0 \mathrm{~Hz}, 1 \mathrm{H}) .{ }^{13} \mathrm{C} \mathrm{NMR}\left(\mathrm{CDCl}_{3}\right): \delta 26.4$, 32.4, 91.6, 110.2, 120.5, 121.1, 125.1, 126.0, 129.4, 137.4, 161.6. IR (nujol): 3380, 1646, $1547 \mathrm{~cm}^{-}$

${ }^{1}$. MS (EI, 70 eV): m/z (\%) 268 (100), 266 (100), 238 (41), 236 (48), 210 (18), 209 (19), 208 (21), 
169 (36), 167 (35), 158 (36), 129 (36), 128 (35), 114 (22), 89 (24), 88 (18). Anal calcd for $\mathrm{C}_{11} \mathrm{H}_{11} \mathrm{BrN}_{2} \mathrm{O}: \mathrm{C}, 49.46 ; \mathrm{H}, 4.15 ; \mathrm{N}, 10.49$. Found: $\mathrm{C}, 49.23 ; \mathrm{H}, 4.02 ; \mathrm{N}, 10.54$.

\section{3-Bromo-benzofuran-2-carboxylic acid methylamide (2e)}

Yield: 87 \%. M.p.: $154-156{ }^{\circ} \mathrm{C}\left(i-\mathrm{Pr}_{2} \mathrm{O}\right) .{ }^{1} \mathrm{H}$ NMR $\left(\mathrm{CDCl}_{3} / \mathrm{D}_{2} \mathrm{O}\right): \delta 3.04(\mathrm{~s}, 3 \mathrm{H}), 7.38-7.43(\mathrm{~m}, 1 \mathrm{H})$, 7.50-7.54 (m, 2H), $7.67(\mathrm{~d}, J=7.8 \mathrm{~Hz}, 1 \mathrm{H}) .{ }^{13} \mathrm{C} \mathrm{NMR}\left(\mathrm{CDCl}_{3}\right): \delta 26.0,101.5,111.8,122.3,124.1$, 128.0, 128.3, 143.2, 153.0, 158.7. IR (nujol): $3375,1661,1581 \mathrm{~cm}^{-1}$; Anal calcd for $\mathrm{C}_{10} \mathrm{H}_{8} \mathrm{BrNO}_{2}$ : C, 47.27; H, 3.17; N, 5.51. Found: C, 47.10; H, 3.14; N, 5.54.

\section{3-Bromo-benzo[b]thiophene-2-carboxylic acid 4-methoxy-benzylamide (2c).}

$\left(\mathrm{R}^{1}=\mathrm{PMB}\right)$ : Yield $95 \%$. Mp: 128-129 ${ }^{\circ} \mathrm{C}(\mathrm{AcOEt}) .{ }^{1} \mathrm{H}$ NMR $\left(\mathrm{CDCl}_{3} / \mathrm{D}_{2} \mathrm{O}\right): \delta 3.81(\mathrm{~s}, 3 \mathrm{H}), 4.65(\mathrm{~s}$, $2 \mathrm{H}), 6.9(\mathrm{~d}, \mathrm{~J}=8.6 \mathrm{~Hz}, 2 \mathrm{H}), 7.33(\mathrm{~d}, \mathrm{~J}=8.6 \mathrm{~Hz}, 2 \mathrm{H}), 7.45-7.51(\mathrm{~m}, 2 \mathrm{H}), 7.81-7.88(\mathrm{~m}, 2 \mathrm{H}) .{ }^{13} \mathrm{C}$ $\mathrm{NMR}\left(\mathrm{CDCl}_{3}\right) \delta 43.8,55.3,106.2,114.2,122.6,124.5,125.5,127.3,129.2,129.6,134.9,138.5$, 138.6, 159.2, 160.7. IR (nujol): 3273, 1635, $1548 \mathrm{~cm}^{-1}$. MS (EI, $70 \mathrm{eV}$ ): m/z (\%) 377 (97), 375 (95), 296 (91), 241 (89), 239 (87), 214 (33), 213 (25), 212 (33), 211 (21), 188 (27), 161 (43), 136 (35), 135 (100), 134 (42), 133 (21), 132 (63), 121 (76), 109 (18), 89 (29), 78 (17), 77 (23). Anal Calcd for $\mathrm{C}_{17} \mathrm{H}_{14} \mathrm{BrNO}_{2} \mathrm{~S}$ : C, 54.27; H, 3.75; N, 3.72. Found: C, 54.43; H, 3.76; N, 3.86.

\section{3-Bromo-benzo[b]thiophene-2-carboxilic acid phenylamide (2c)}

$\left(\mathrm{R}^{1}=\mathrm{Ph}\right.$ ): Yield 91\%. Mp: 157-158 ${ }^{\circ} \mathrm{C}$ (AcOEt). IR (nujol): 3305, 1641, $1542 \mathrm{~cm}^{-1} ;{ }^{1} \mathrm{H}$ NMR $\left(\mathrm{CDCl}_{3} / \mathrm{D}_{2} \mathrm{O}\right): 7.19(\mathrm{t}, \mathrm{J}=7.4 \mathrm{~Hz}, 1 \mathrm{H}), 7.4(\mathrm{t}, \mathrm{J}=7.8 \mathrm{~Hz}, 2 \mathrm{H}), 7.49-7.55(\mathrm{~m}, 2 \mathrm{H}), 7.7$ (d, J = 8.1 Hz, 2H), 7.84-7.94 (m, 2H). ${ }^{13} \mathrm{C}$ NMR (rotameric mixture) $\delta 106.3,120.3,122.6,122.8,123.2$, 124.6, 125.0, 125.5, 125.6, 127.5, 129.1, 135.3, 137.1, 138.4, 138.7, 158.7. MS (EI, 70 eV): m/z (\%) 333 (17), 331 (16), 252 (43), 241 (100), 239 (97), 213 (18), 211 (19), 132 (53), 65 (20). Anal calcd for $\mathrm{C}_{15} \mathrm{H}_{10}$ BrNOS: C, 54.23; H, 3.03; N, 4.22. Found: C, 54.28; H, 3.20; N, 3.90.

Synthesis of 1. A Schlenk-type flask was charged under nitrogen with $\mathrm{Pd}(\mathrm{OAc})_{2}(5.04 \mathrm{mg}, 0.0225$ mmol), tri-2-furylphosphine (10.4 mg, $0.045 \mathrm{mmol}), \mathrm{K}_{2} \mathrm{CO}_{3}(125 \mathrm{mg}, 0.90 \mathrm{mmol}), 2$ (0.45 mmol) and anhydrous DMF $(10 \mathrm{~mL})$. The reaction mixture was heated under stirring at $105{ }^{\circ} \mathrm{C}$ for the time reported in Table 1, then cooled to $\mathrm{r}$. t.. The reaction was quenched with saturated aq. $\mathrm{NH}_{4} \mathrm{Cl}$ (75 $\mathrm{mL})$ and the aqueous layer was extracted with AcOEt $(3 \mathrm{X} 40 \mathrm{~mL})$. The combined organic extracts were washed with brine, separated and dried over $\mathrm{Na}_{2} \mathrm{SO}_{4}$. Removal of the solvent under reduced pressure gave a solid material which was purified by flash chromatography on silica gel (petroleum ether/AcOEt).

\section{5-Methyl-5H-phenanthridin-6-one (1a)}




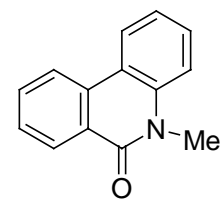

Yield: $23 \%$, mp $106-107^{\circ} \mathrm{C}$ (hexane) (lit. ${ }^{8} \mathrm{mp} 108.5^{\circ} \mathrm{C}$ ).

4-Methyl-4H-3,6-dithia-4-azaindacen-5-one (2b).

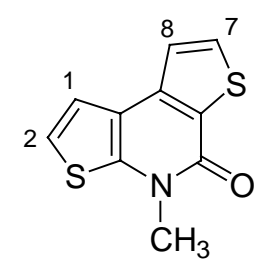

Yield: $30 \%$. M.p.: $133-134^{\circ} \mathrm{C}(i \mathrm{PrOH}) .{ }^{1} \mathrm{H} \mathrm{NMR}\left(\mathrm{CDCl}_{3}\right): \delta 3.80\left(\mathrm{~s}, \mathrm{CH}_{3}, 3 \mathrm{H}\right), 7.07(\mathrm{~d}, J=5.6 \mathrm{~Hz}$, $\mathrm{H} 2,1 \mathrm{H}), 7.38(\mathrm{~d}, J=5.6 \mathrm{~Hz}, \mathrm{H} 1,1 \mathrm{H}), 7.46(\mathrm{~d}, J=5.2 \mathrm{~Hz}, \mathrm{H} 8,1 \mathrm{H}), 7.77$ (d, $J=5.2 \mathrm{~Hz}, \mathrm{H} 7,1 \mathrm{H})$; ${ }^{13} \mathrm{C} \mathrm{NMR}\left(\mathrm{CDCl}_{3}\right): \delta 33.84\left(\mathrm{CH}_{3}\right), 116.67(\mathrm{C} 2), 117.29(\mathrm{C} 8 \mathrm{~b}), 121.99(\mathrm{C} 1), 122.25(\mathrm{C} 8), 127.86$ (C5a), 133.95 (C7), 140.46 (C8a), 145.32 (C3a), 158.05 (C5). IR (nujol): 1645, $1629 \mathrm{~cm}^{-1}$. MS m/z 221 (100), 206 (10), 192 (26), 178 (20). Anal.Calcd. for $\mathrm{C}_{10} \mathrm{H}_{7} \mathrm{NOS}_{2}$ : C, 54.27; H, 3.19; N, 6.33. Found: C, 54.37; H, 3.22; N, 6.34.

6-Methyl-6H-5,8-dithia-6-azaindeno[2,1-c]fluoren-7-one (1c).

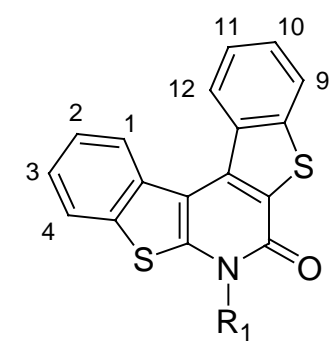

$\left(\mathrm{R}^{1}=\mathrm{Me}\right)$ : Yield: $75 \%$. M.p.: 213-215 ${ }^{\circ} \mathrm{C}(\mathrm{AcOEt}) .{ }^{1} \mathrm{H} \mathrm{NMR}\left(\mathrm{CDCl}_{3}\right): \delta 3.73\left(\mathrm{~s}, \mathrm{CH}_{3}, 3 \mathrm{H}\right), 7.29(\mathrm{t}$, $J=7.6 \mathrm{~Hz}, \mathrm{H} 3,1 \mathrm{H}), 7.39$ and $7.42(2 \mathrm{t}, \mathrm{H} 2$ and H11, 2H), $7.51(\mathrm{dd}, J=7.5,7.1 \mathrm{~Hz}, \mathrm{H} 10,1 \mathrm{H}), 7.73$ (d, $J=7.8 \mathrm{~Hz}, \mathrm{H} 4,1 \mathrm{H}), 7.92$ (d, $J=7.9 \mathrm{~Hz}, \mathrm{H} 9,1 \mathrm{H}), 8.37$ (d, $J=8.1 \mathrm{~Hz}, \mathrm{H} 1,1 \mathrm{H}), 8.58$ (d, $J=8.1$ $\mathrm{Hz}, \mathrm{H} 12,1 \mathrm{H}) ;{ }^{13} \mathrm{C} \mathrm{NMR}\left(\mathrm{CDCl}_{3}\right): \delta 34.01\left(\mathrm{CH}_{3}\right), 112.30(\mathrm{C} 12 \mathrm{c}), 122.51(\mathrm{C} 4), 123.39(\mathrm{C} 1), 123.58$ (C9), 123.94 (C3), 124.10 (C11), 124.94 (C2), 126.31 (C12), 127.40 (C10), 128.74 (C7a), 133.07 (C4a), 134.50 (C12d), 134.77 (C12a), 136.55 (C12b), 142.73 (C8a), 146.44 (C5a), 158.04 (C7). IR (nujol): $1637 \mathrm{~cm}^{-1}$. MS m/z 321 (100), 292 (10), 278 (20), 264 (5). Anal. Calcd. for $\mathrm{C}_{18} \mathrm{H}_{11} \mathrm{NOS}_{2}$ : C, 67.26; H, 3.45; N, 4.36. Found: C, 67.24; H, 4.32; N, 4.63.

6-(4-Methoxybenzyl)-6H-5,8-dithia-6-azaindeno[2,1-c]fluoren-7-one (1c).

$\left(\mathrm{R}^{1}=\mathrm{PMB}\right):$ Yield: $50 \%$. Mp: $211-213{ }^{\circ} \mathrm{C}\left(\mathrm{AcOEt} / \mathrm{CH}_{2} \mathrm{Cl}_{2}\right.$ 9/1). ${ }^{1} \mathrm{H} \mathrm{NMR}\left(\mathrm{CDCl}_{3}\right): \delta 3.75$ (s, 3H), $5.54(\mathrm{~s}, 2 \mathrm{H}), 6.82-6.87(\mathrm{~m}, 2 \mathrm{H}), 7.38(\mathrm{t}, J=7.7 \mathrm{~Hz}, 1 \mathrm{H}), 7.43-7.48(\mathrm{~m}, 2 \mathrm{H}), 7.50-7.62(\mathrm{~m}, 3 \mathrm{H}), 7.83$ $(\mathrm{d}, J=7.9 \mathrm{~Hz}, 1 \mathrm{H}), 8.04(\mathrm{~d}, J=7.2 \mathrm{~Hz}, 1 \mathrm{H}), 8.75(\mathrm{~d}, J=7.5 \mathrm{~Hz}, 1 \mathrm{H}), 8.86(\mathrm{~d}, J=7.3 \mathrm{~Hz}, 1 \mathrm{H}) .{ }^{13} \mathrm{C}$ 
NMR $\left(\mathrm{CDCl}_{3}\right): \delta 50.6,55.2,113.0,114.1,122.6,123.5,123.7,124.1,124.2,124.9,126.5,126.8$, 127.6, 129.7, 133.5, 134.4, 135.0, 137.0, 143.1, 146.0, 158.4, 159.5. IR (nujol): $1640 \mathrm{~cm}^{-1}$. HRMS (ESI) Calcd for $\mathrm{C}_{25} \mathrm{H}_{17} \mathrm{NO}_{2} \mathrm{~S}_{2} \mathrm{Na}: 450.05929$. Found: 450.05816 .

\section{6-(Phenyl)-6H-5,8-dithia-6-azaindeno[2,1-c]fluoren-7-one (1c).}

$\left(\mathrm{R}^{1}=\mathrm{Ph}\right)$ : Yield 90\%: Mp: 252-253 ${ }^{\circ} \mathrm{C}(\mathrm{AcOEt}) ;{ }^{1} \mathrm{H} \mathrm{NMR}\left(\mathrm{CDCl}_{3}\right): \delta 7.37(\mathrm{t}, \mathrm{J}=7.7 \mathrm{~Hz}, 1 \mathrm{H}), 7.52-$ $7.68(\mathrm{~m}, 8 \mathrm{H}), 7.73(\mathrm{~d}, \mathrm{~J}=8.0 \mathrm{~Hz}, 1 \mathrm{H}), 8.03-8.08(\mathrm{~m}, 1 \mathrm{H}), 8.66(\mathrm{~d}, \mathrm{~J}=8.2 \mathrm{~Hz}, 1 \mathrm{H}), 8.88-8.94(\mathrm{~m}$, $1 \mathrm{H}) ;{ }^{13} \mathrm{C} \mathrm{NMR}\left(\mathrm{CDCl}_{3}\right): \delta 112.8,122.5,123.6,123.8,124.2,124.3,125.0,126.6,127.7,127.9$, 130.0, 130.2, 133.9, 134.6, 135.1, 137.5, 138.8, 143.2, 148.0, 158.2; IR (nujol) 1651: $\mathrm{cm}^{-1}$; HRMS (ESI) Calcd for $\mathrm{C}_{23} \mathrm{H}_{13} \mathrm{NOS}_{2} \mathrm{Na}$ : 406.03308. Found: 406.03164.

5,6,8-Trimethyl-6,8-dihydro-5H-5,6,8-triazaindeno[2,1-c]fluoren-7-one (1d).

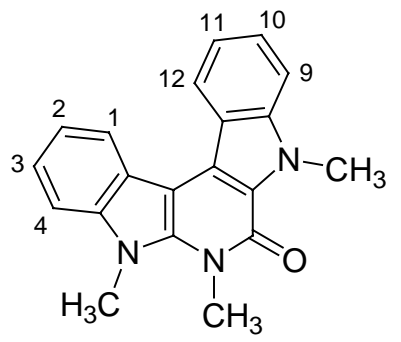

Yield: 70\%. M.p.: $257-258{ }^{\circ} \mathrm{C}$ with decomp. $\left(\mathrm{AcOEt} / \mathrm{CH}_{2} \mathrm{Cl}_{2}\right.$ 9/1). ${ }^{1} \mathrm{H}$ NMR (DMSO- $\left.d_{6}\right): \delta 4.04$ $\left(3 \mathrm{H}, \mathrm{s}, \mathrm{CH}_{3} \mathrm{~N}(6)\right), 4.12\left(3 \mathrm{H}, \mathrm{s}, \mathrm{CH}_{3} \mathrm{~N}(5)\right), 4.26\left(3 \mathrm{H}, \mathrm{s}, \mathrm{CH}_{3} \mathrm{~N}(8)\right), 7.26-7.37(3 \mathrm{H}, \mathrm{m}, \mathrm{H} 3, \mathrm{H} 2, \mathrm{H} 11)$, $7.54(1 \mathrm{H}, \mathrm{dd}, J=8.1 .7 .1 \mathrm{~Hz}, \mathrm{H} 10), 7.58-7.70$ (2H, m, H4, H9), 8.31-8.38 (1H, m, H1), 8.58 (1H, br $\mathrm{d}, J=8.1 \mathrm{~Hz}, \mathrm{H} 12) ;{ }^{13} \mathrm{C} \mathrm{NMR}\left(\mathrm{DMSO}-d_{6}\right): \delta 31.07\left(\mathrm{CH}_{3} \mathrm{~N}(8)\right), 31.76\left(\mathrm{CH}_{3} \mathrm{~N}(6)\right), 33.55\left(\mathrm{CH}_{3} \mathrm{~N}(5)\right)$, 96.01 (C12c), 110.19 (C4), 110.55 (C9), 119.57 (C11, C12b), 119.71 (C1), 119.94 (C12a), 120.47 (C2), 121.36 (C12d), 121.49 (C3), 121.61 (C7a), 123.73 (C12), 126.22 (C10), 137.35 (C4a), 138.33 (C5a), 140.78 (C8a), 155.15 (C7); IR (nujol): $1651 \mathrm{~cm}^{-1}$. MS (EI, 70 eV): m/z (\%) 315 (100), 300 (59), 257 (12), 157 (17), 150 (15). Anal.Calcd for $\mathrm{C}_{20} \mathrm{H}_{17} \mathrm{~N}_{3} \mathrm{O}: \mathrm{C}, 76.17 ; \mathrm{H}, 5.43 ; \mathrm{N}, 13.32$. Found: C, 76.14; H, 5.39; N, 13.39 .

\section{6-Methyl-6H-5,8-dioxa-6-azaindeno[2,1-c]fluoren-7-one (1e)}

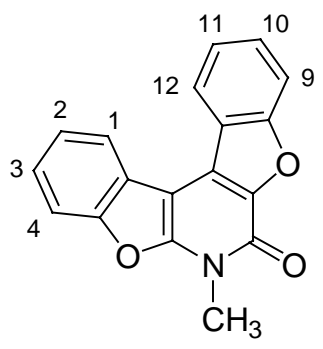

Yield: 52\%. M.p.: $246-248{ }^{\circ} \mathrm{C}\left(\mathrm{AcOEt} / \mathrm{CH}_{2} \mathrm{Cl}_{2}\right.$ 9/1). ${ }^{1} \mathrm{H} \mathrm{NMR}\left(\mathrm{CDCl}_{3}\right): \delta 3.88\left(\mathrm{~s}, \mathrm{CH}_{3}, 3 \mathrm{H}\right), 7.34$ (ddd, $J=7.7,7.4,1.4 \mathrm{~Hz}, \mathrm{H} 3,1 \mathrm{H}), 7.41$ and 7.45 (2td, H2 and H11, 2H), 7.55 and 7.59 (br d, H4 and td, H10, 2H), 7.69 (dt, $J=8.4,0.9 \mathrm{~Hz}, \mathrm{H} 9,1 \mathrm{H}), 7.96$ (d further split, $J=7.7 \mathrm{~Hz}, \mathrm{H1}, 1 \mathrm{H}), 8.16$ (d further split, $J=7.7 \mathrm{~Hz}, \mathrm{H} 12,1 \mathrm{H}) ;{ }^{13} \mathrm{C} \mathrm{NMR}\left(\mathrm{CDCl}_{3}\right): 29.14\left(\mathrm{CH}_{3}\right), 93.75(\mathrm{C} 12 \mathrm{c}), 111.53(\mathrm{C} 4)$, 
113.08 (C9), 120.21 (C1), 122.68 (C12a), 123.41 (C12), 123.63 (C11), 123.90 and 123.96 (C12b and C12d), 124.02 (C3), 124.34 (C2), 129.06 (C10), 140.52 (C7a), 150.41 and 152.04 (C5a and C7), 152.15 (C4a), 157.00 (C8a); IR (nujol): $1672 \mathrm{~cm}^{-1}$. HRMS (ESI) Calcd for $\mathrm{C}_{18} \mathrm{H}_{11} \mathrm{NO}_{3} \mathrm{Na}$ : 312.06311; Found: 312.06284.

Characteristic data of 7-[(2-Methylaminocarbonyl)phenyl]-5-methyl-5H-phenanthridin-6-one (3)

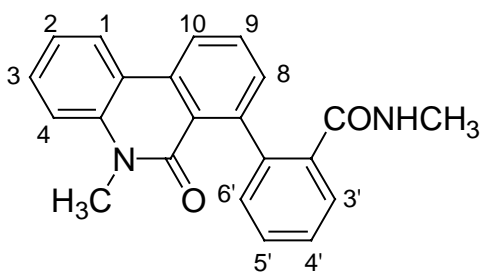

Yield 34\%; Mp $186^{\circ} \mathrm{C}$ (hexane); ${ }^{1} \mathrm{H}$ NMR $\left(\mathrm{CDCl}_{3}\right): \delta 2.53\left(\mathrm{~d}, J=5.0 \mathrm{~Hz}, \mathrm{CH}_{3} \mathrm{NH}, 3 \mathrm{H}\right), 3.72$ (s, $\mathrm{CH}_{3} \mathrm{~N}, 3 \mathrm{H}$ ), 6.65 (br q, $\left.J=4.8 \mathrm{~Hz}, \mathrm{NH}, 1 \mathrm{H}\right), 7.00-6.95$ (m, H6', 1H), 7.32 (dd, $J=7.4,1.1 \mathrm{~Hz}, \mathrm{H} 8$, 1H), 7.46-7.34 (m, H2, H4, H4', H5', 4H), 7.60 (ddd, $J=8.6,7.1,1.5$ Hz, H3, 1H), 7.75-7.65 (m, H9, H3', 2H), 8.35 and $8.37(2 \mathrm{dd}, J=8.4,1.1 \mathrm{~Hz}, J=8.1,1.5 \mathrm{~Hz}, \mathrm{H} 10$ and $\mathrm{H} 1,2 \mathrm{H}) ;{ }^{13} \mathrm{C}$ NMR $\left(\mathrm{CDCl}_{3}\right): \delta 26.21\left(\mathrm{CH}_{3} \mathrm{NH}\right), 30.18\left(\mathrm{CH}_{3} \mathrm{~N}\right), 115.07(\mathrm{C} 4), 119.34(\mathrm{C} 10 \mathrm{~b}), 121.53(\mathrm{C} 10), 122.87$ (C5’), 123.05 (C6a), 123.74 (C1), 126.97 (C4’), 127.45 (C3’), 128.41 (C6’), 129.02 (C2), 129.93 (C3), 131.29 (C8), 131.66 (C9), 134.80 (C10a, C2'), 137.94 (C4a), 141.38 (C1'), 143.18 (C7), 162.22 (C6), 170.85 (CO(C2')); IR (KBr, cm $\left.{ }^{-1}\right): v 3376,1641$; MS (EI, $\left.70 \mathrm{eV}\right): \mathrm{m} / \mathrm{z}(\%) 343$ (67, $\mathrm{M}+1$ ), 312 (100), 284 (80), 269 (20), 154 (55); Anal calcd for $\mathrm{C}_{22} \mathrm{H}_{18} \mathrm{~N}_{2} \mathrm{O}_{2}$ : C, 77.17; H, 5.30; N, 8.18. Found: C, 77.37; H, 5.38; N, 8.23.

Characteristic data of 2-[3-(2-Methylaminocarbonyl)thienyl]-4-methyl-4H-3,6-dithia-4azaindacen-5-one (4).

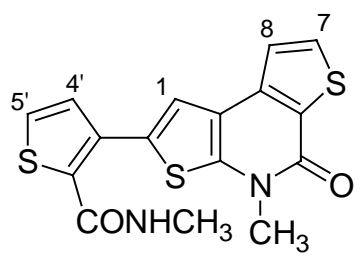

Yield: $20 \%$. M.p.: $249-250{ }^{\circ} \mathrm{C}$, with decomposition (AcOEt). ${ }^{1} \mathrm{H}$ NMR $\left(\mathrm{CDCl}_{3}\right): \delta 2.94(\mathrm{~d}, J=4.9$ $\left.\mathrm{Hz}, \mathrm{CH}_{3} \mathrm{NH}, 3 \mathrm{H}\right) ; 3.82$ (s, $\left.\mathrm{CH}_{3} \mathrm{~N}, 3 \mathrm{H}\right), 6.08$ (br s, NH, 1H), 7.20 (d, J = 5.1 Hz, H4', 1H), 7.47 and $7.50\left(2 \mathrm{~d}, J=5.6 \mathrm{~Hz}, J=5.2 \mathrm{~Hz}, \mathrm{H} 5^{\prime}\right.$ and $\left.\mathrm{H} 8,2 \mathrm{H}\right), 7.63(\mathrm{~s}, \mathrm{H} 1,1 \mathrm{H}), 7.82(\mathrm{~d}, J=5.2 \mathrm{~Hz}, \mathrm{H} 7,1 \mathrm{H})$; ${ }^{13} \mathrm{C}$ NMR $\left(\mathrm{CDCl}_{3}\right): \delta 26.92\left(\mathrm{CH}_{3} \mathrm{NH}\right), 33.89\left(\mathrm{CH}_{3} \mathrm{~N}\right), 117.41(\mathrm{C} 8 \mathrm{~b}), 122.22(\mathrm{C} 8), 122.37(\mathrm{C} 1)$, 127.55 (C2), 127.80 (C5'), 128.01 (C5a), 130.78 (C4'), 133.17 (C3'), 133.24 (C2'), 134.29 (C7), 140.26 (C8a), 146.04 (C3a), 157.87 (C5), 162.72 (CO (C2')). IR (nujol): 3267, 1650, $1615 \mathrm{~cm}^{-1}$. 
MS m/z 360 (100), 330 (10), 302 (8), 287 (5). Anal.Calcd. for $\mathrm{C}_{16} \mathrm{H}_{12} \mathrm{~N}_{2} \mathrm{O}_{2} \mathrm{~S}_{3}: \mathrm{C}, 53.31 ; \mathrm{H}, 3.36$; , 7.77. Found: C, 53.43; H, 3.45; N, 7.79.

Characteristic data of [3,3']bi[benzo[b]thiophenyl]-2,2'-dicarboxylic acid bis-(4-methoxybenzyl amide).

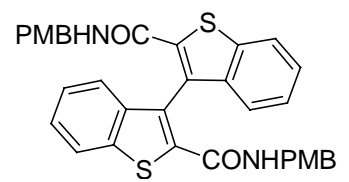

${ }^{1} \mathrm{H}$ NMR (DMSOd $\left.6,300 \mathrm{MHz}\right): \delta 3.7$ (s, 6H), 4.10 (dd, J = 15.1, 5.3 2H), $4.3(\mathrm{dd}, \mathrm{J}=15.1,6.5 \mathrm{~Hz}$, 2H), 6.59-6.72 (m, 8H), $7.12(\mathrm{~d}, \mathrm{~J}=8.0 \mathrm{~Hz}, 2 \mathrm{H}), 7.35(\mathrm{t}, \mathrm{J}=7.6 \mathrm{~Hz}, 2 \mathrm{H}), 7.53(\mathrm{t}, \mathrm{J}=7.6 \mathrm{~Hz}, 2 \mathrm{H})$, 8.17 (d, J = 8.0 Hz, 2H), 8.73 (br t, 2H). MS/EI m/z $592\left(\mathrm{M}^{+}, 70\right), 336$ (30), 293 (100).

Reaction of biphenyl-2,2'-dicarboxylic acid bis-phenylamide ${ }^{9}$ in the presence of $\mathrm{PdCl}_{2}\left(\mathrm{CH}_{3} \mathrm{CN}\right)_{2}$. A Schlenck-type flask was charged under nitrogen with $\mathrm{PdCl}_{2}\left(\mathrm{CH}_{3} \mathrm{CN}\right)_{2}(16 \mathrm{mg}, 0.062 \mathrm{mmol})$, $\mathrm{K}_{2} \mathrm{CO}_{3}(108 \mathrm{mg}, 0.78 \mathrm{mmol})$, bis-amide $(25 \mathrm{mg}, 0.064 \mathrm{mmol})$ and anhydrous DMF $(10 \mathrm{~mL})$. The reaction mixture was heated under stirring at $105^{\circ} \mathrm{C}$. After two hours palladium black separated out and the reaction mixture was diluted with $\mathrm{CH}_{2} \mathrm{Cl}_{2}(10 \mathrm{~mL})$ and treated with water. After conventional work-up the GC and GC-MS analyses of the crude showed the presence of unreacted diammide (92\%) together with 6H-5-phenyl-phenanthridin-6-one (ca. 5\%), which was compared with a sample prepared according to a literature procedure for the corresponding methyl derivative. ${ }^{10,11}$

NMR assignments for compounds $\mathbf{1 c}, \mathbf{3}$ and $\mathbf{4}$.

The reported assignments are based on decoupling and 2D experiments. The regiochemistry of compounds 1, 3 and $\mathbf{4}$ was established from the NOESY (Nuclear Overhauser Effect SpectroscopY) spectra. For compound 1c, taken as an example, the presence of a strong NOE interaction between the two doublets at low field (8.58 and 8.37, respectively) belonging to two aromatic moieties, allows to identify $\mathrm{H} 1$ and $\mathrm{H} 12$ and is in agreement with the assigned structure where the two sulfur atoms are placed on the same side of the aminocarbonyl group. Confirmation of the structure comes from long range C-H correlation HMQC (Heteronuclear Multiple-Quantum Coherence) experiments which also allow complete assignments of quaternary carbons. As expected, the methyl protons correlate with C7 and C5a which can be distinguished by chemical shifts ( $\delta 158.04$ for the carbonyl C7 and 146.44 for C5a, $\alpha$ to nitrogen). The assignment of C7a is straightforward since it is the only carbon without any $\mathrm{C}-\mathrm{H}$ correlation. The four ring-junction quaternary carbons $\mathrm{C} 4 \mathrm{a}, \mathrm{C} 8 \mathrm{a}$, $\mathrm{C} 12 \mathrm{a}$ and $\mathrm{C} 12 \mathrm{~d}$ can be readily recognized because each of them shows a long-range correlation 
with two aromatic protons while the remaining ones, $\mathrm{C} 12 \mathrm{~b}$ and $\mathrm{C} 12 \mathrm{c}$, correlate only with one proton each. $\mathrm{C} 12 \mathrm{c}$ is distinguished from $\mathrm{C} 12 \mathrm{~b}$ on the basis of the ca. $24 \mathrm{ppm}$ difference between their resonances and is assigned to the higher field signal (112.30) since it is $\beta$ to nitrogen. The assignments of $\mathrm{C} 12 \mathrm{~b}$ and $\mathrm{C} 12 \mathrm{c}$ allow to differentiate $\mathrm{H} 1$ and $\mathrm{H} 12$ thus completing both the carbon and proton assignments.

For compound $\mathbf{3}$ both proton and carbon assignments are quite straightforward. The noesy spectra show a dipolar interaction between the methyl protons, bonded to nitrogen of the condensed structure, and a doublet at ca. $\delta 7.43$. This interaction, complemented by the other cross peaks and by the result of decoupling experiments, allow to assign $\mathrm{H} 4$ and the other protons thus confirming that the aminocarbonylphenyl unit is situated on the side of the $\mathrm{CO}$ group of the condensed structure. The long-range correlations observed in the HMQC spectra are in agreement with the assigned formula.

For compound 4 the singlet at $\delta 7.63$ shows NOE interactions with the broad signal at $\delta 6.12(\mathrm{NH})$ and with two doublets at $\delta 7.50$ and 7.20, respectively. The former corresponds to a proton of a condensed thiophene unit while the latter pertains to the methylaminocarbonylthienyl group. These interactions allow to assign $\mathrm{H} 1$ and to locate the free thienyl ring $\alpha$ to a sulfur atom. Confirmation of the assigned structure derives from HMQC experiments. Analogously to compounds $\mathbf{1}$ the methyl protons of $\mathrm{N}(4)$ correlate with $\mathrm{C} 3 \mathrm{a}$ and $\mathrm{C} 5$ which are discriminated on the basis of their chemical shifts. C3a correlates only with the singlet at $\delta 7.63$ (H1). This observation complements the noesy interactions.

\section{References}

1. Avaible from Aldrich and Fluka Chemicals.

2. Ohkata, K.; Ohnishi, M.; Akiba, K. Tetrahedron Lett. 1988, 42, 5401-5404.

3. Ferraccioli, R., Carenzi, D., Rombolà, O., Catellani, M. Organic Lett. 2004, 6, 4759-4762.

4. Konuri, M. Chem Abs, 1960, 1487.

5. Liu, Y.; Gribble, G. W. Tetrahedron Lett.; 43; 2002; 7135-7138.

6. Benincori, T; Brenna, E.; Sannicolò, F.; Trimarco, L.; Antognazza, P.; Cesarotti, E.; Demartin, F; Pilati, T. J. Org. Chem. 1996, 61, 6244-6251.

7. Cugnon de Sevicourt M., Robba, M. Bull. Soc. Chim. France, 1977, 139-141.

8. Bowman, W. R., Heaney, H., Jordan, B. M. Tetrahedron, 1991, 10119-10128.

9. Roberts, R. C.; T.B. Johnson, T. B. J. Am. Chem. Soc. 1925, 47, 1396.

10. Ames, D. E.; Opalko, A. Tetrahedron 1984, 40, 1919.

11. Datta, I.; Kumar Das T.; Ghosh, S. Tetrahedron 1990, 46, 6821. 